\title{
SISTEMA DE EVALUACIÓN CUALITATIVA A TRAVÉS DE DISPOSITIVOS MÓVILES
}

\author{
José Eucario Parra Castrillón \\ Universidad de San Buenaventura \\ eucarioparra5@gmail.com
}

(Tipo de Artículo: Investigación. Recibido el 16/10/2012. Aprobado el 22/12/2012)

\section{RESUMEN}

El Sistema PruebaL es un producto del proyecto Evaluación Cualitativa a Través de Dispositivos Móviles, cuyo objetivo es analizar e implementar un sistema para gestionar procesos de evaluación.

La temática se enmarca dentro de los conceptos de mobile learnig y su desarrollo consistió en el análisis de requerimientos funcionales y no funcionales, la definición del sistema, el diseño para las relaciones modulares de profesor, estudiante, administrador y pruebas y la implementación utilizando tecnología de teléfonos celulares.

Se alcanzaron dos logros fundamentales: un modelo de evaluación cualitativa automatizada con el sistema PruebaL y la notificación efectiva a los teléfonos celulares matriculados en las bases de datos de estudiantes, para la presentación de las evaluaciones y el acceso Web a estas.

\section{Palabras clave}

Dispositivos móviles , m-learning, escala de Likert, evaluación cualitativa, redes inalámbricas,

\section{QUALITATIVE ASSESSMENT SYSTEM THROUGH MOBILE DEVICES}

\section{ABSTRACT}

The System PruebaL is a product of the research project called Quality Assessment Through Mobile Devices, which aims to analyze and implement a system to manage the evaluation processes.

The subject is in the context of mobile learning concepts and the approach is made through the analysis of functional and nonfunctional requirements, system definition, and design for modular relationships for professor, student, administrator and testing and implementation using cell phone technology.

There are two significant achievements: an automated qualitative assessment model with the system PruebaL and the effective notification for cell phones registered in student databases for conducting assessments and the Web access to them.

\section{Keywords}

Mobile devices, m-learning, Likert scale, qualitative assessment, wireless networks.

\section{SYSTĖME D’EVALUATION QUALITATIVE À TRAVERS DE DISPOSITIFS MOBILES}

\author{
Résumé \\ Le système Pruebal est un produit du projet Evaluation Qualitative à Travers de Dispositifs Mobiles, qu'a comme objectif \\ d'analyser et d'implémenter un système pour gérer des processus d'évaluation. \\ Le thème se situe autour des concepts d'apprentissage sur mobile et est développé à partir de l'analyse des requêtes \\ fonctionnelles et non-fonctionnelles, la définition du système, la conception des rapports modulaires du professeur, étudiante, \\ administrateur et des essais \& implémentation en utilisant technologie de téléphones portables.
}

Il y a deux réussites fondamentales : un model d'évaluation qualitative automatisé avec le système PruebaL et la notification effectif aux téléphones portables enregistrés dans las bases de données d'étudiants, par rapport à la présentation des exams et accès pour le Web.

\section{Mots-clés}

Dispositifs mobiles, apprentissage sur mobile, échelle de Likert, évaluation qualitative, réseaux sans fils. 


\section{INTRODUCCIÓN}

El proyecto contribuye a la investigación aplicada en el campo del Mobile learning (m-learning) y dentro de sus objetivos están crear un estado del arte acerca del desarrollo de aplicativos y proyectos para m-learning que utilicen software libre; determinar en cuáles dispositivos móviles es utilizable el aplicativo desarrollado, mediante una clasificación valorativa a las tecnologías existentes y desarrollar las fases de análisis, diseño, implementación y pruebas de un sistema de m-learning para dispositivos móviles.

Mobile learning es una forma de aprendizaje generada a partir de la conjunción entre el e-learning y la aplicación de los dispositivos móviles (smart devices) inteligentes, como PDAs, smartphones, Ipods, pocket PCs, teléfonos. Se fundamenta en la posibilidad que brindan estos móviles para combinar la movilidad geográfica con la virtual, lo que permite el aprendizaje en el momento en que se necesita, en el lugar donde se encuentre y con la información precisa que se requiera.

El m-learning se desarrolla apoyado en las siguientes características de los servicios móviles [1]: accesibilidad, conveniencia, inmediatez, localización, personalización y ubicuidad.

En su esencia el proyecto crea un servicio de formulario para la Web, donde el profesor diseña la estructura de la prueba, la cual consiste en la escritura de los enunciados de las preguntas, la escritura de las opciones de respuesta para la homologación con la escala de Likert y el tiempo de respuesta. Las preguntas se plantean para que el estudiante responda desde su dispositivo móvil a unas opciones, las que internamente serán interpretadas por el software para la clasificación en un modelo de Escala de Likert [2].

\section{DESARROLLO DEL M-LEARNING}

El e-learning o aprendizaje electrónico es más que un sistema de acceso a información y de distribución de conocimientos. Además de estas dos funciones, debe proveer mecanismos de participación pedagógica para la interacción entre los participantes, teniendo en cuenta la educación como conjunto de procesos constructivos, la tecnología como soporte y medio para la creación de ambientes educativos y la comunicación como elemento esencial para el reconocimiento y vinculación de las personas. El e-learning facilita el acceso al aprendizaje en función de las necesidades del usuario, en el lugar que lo requiera.

Acorde con los avances de la tecnología, el e-learning se ha venido transformando. Es así como las clases digitales venían antes integradas en $C D$ con un buen nivel de multimedia, pero sin posibilidad de interacciones de los usuarios con el profesor y con la dificultad de poder actualizar los contenidos. Luego con la disponibilidad de Internet, la fuerza la toman los cursos on-line, con la posibilidad de interacción entre los participantes, la versatilidad de las comunicaciones sincrónicas o asincrónicas, la actualización y adaptación de los contenidos y la utilización de plataformas LMS -Learning Management System-, para la gestión de los aprendizajes. Terminado la primera década del siglo XXI, con el advenimiento de la tecnología móvil, el desarrollo del e-learning viene adquiriendo la forma de m-learning, acorde con la masificación de los dispositivos móviles como recursos que la gente utiliza para comunicarse y para acceder a información en distintos formatos.

Mobile learning (m-learning) es una forma de aprendizaje generada a partir de la conjunción entre el e-learning y la aplicación de los dispositivos móviles (smart devices) inteligentes, como los teléfonos celulares. Se fundamenta en la posibilidad que brindan estos móviles para combinar la movilidad geográfica con la virtual, lo que permite el aprendizaje en el momento en que se necesita, en el lugar donde se encuentre el usuario y con la información precisa que se requiera.

El m-learning se desarrolla apoyado con características de los servicios móviles como los siguientes [1]: accesibilidad (tecnológicamente son pocas las limitaciones en el tiempo o en el espacio para utilizar los servicios y comunicarse), conveniencia (los servicios se utilizan dónde y cuándo se quiere), inmediatez (el acto se comunica en tiempo real), personalización (los servicios y los terminales son adaptables a las necesidades y gustos de los usuarios) y ubicuidad.

También se formulan las siguientes ventajas funcionales del m-learning [1]:

- Aprendizaje en todo tiempo y en cualquier lugar: No se requiere estar en un lugar particular ni a una hora determinada para aprender. El dispositivo móvil puede ser usado en cualquier lugar, por lo que el aprendizaje se personaliza y adapta a los requerimientos y disponibilidades individuales de cada persona.

- Los dispositivos móviles posibilitan la interacción en tiempo real entre estudiantes y profesor. Se facilita de forma automática la retroalimentación por parte del profesor.

- Mayor cobertura: La telefonía móvil está al alcance de la generalidad de la población. El dispositivo móvil es un accesorio cotidiano.

- Mayor accesibilidad. La realidad tecnológica es que los dispositivos móviles tengan conexiones a las redes y servicios de acceso a la Web.

- Mayor portabilidad y funcionalidad: Los estudiantes pueden escribir anotaciones directamente en el dispositivo, durante el mismo tiempo que recibe los objetos de aprendizaje y las comunicaciones con sus asesores. Además, puede grabar para luego acceder a las instrucciones. 
- Aprendizaje colaborativo. La tecnología móvil favorece que los estudiantes puedan compartir el desarrollo de ciertas actividades con distintos compañeros, creando equipos, socializando, compartiendo respuestas, compartiendo propuestas.

- Se facilita el aprendizaje exploratorio: En tiempo real, en la medida que se recibe información se puede estar explorando, experimentando y aplicando sobre contextos y campos de práctica.

El m-learning es una extensión del e-learning y por esto los análisis e investigaciones deben considerar no solo la capacidad tecnológica del dispositivo, sino que acuerdo al siguiente listado de posibles limitaciones, se deben considerar aspectos como:

- Las intenciones pedagógicas deben tener un alcance relacionado con las limitaciones de la multimedia interactiva en los dispositivos móviles.

- Características como la usabilidad, la visibilidad y la navegabilidad deben acoplarse a las condiciones del tamaño reducido de los dispositivos.

- La intensidad de la comunicación, el volumen de los objetos, la extensión de los mensajes, deben considerarse teniendo en cuenta las limitaciones de los dispositivos.

Un aporte importante sobre la incidencia en la relación tecnología - pedagogía, se hace en [3]. Se concluye como los dispositivos móviles conllevan a cambios en los componentes de diseño y enseñanza de los ambientes virtuales. "ya que la incorporación de dispositivos de mobile learning "obliga" a pensar de forma diferente, a visualizar los contenidos, los materiales y las estrategias de distinto modo cuando se visualiza que el alumno estará interactuando en diversos espacios y escenarios".

Además, se plantea en este mismo artículo que la comunicación entre los participantes en el ambiente de aprendizaje virtual con m-learning se incrementa, dada la convergencia de distintos canales y las características de mensajería instantánea que fácilmente allí se adopta. Se incrementa también la posibilidad del contacto bidireccional en forma sincrónica y de descargar los objetos de aprendizaje para reproducirlos posteriormente en cualquier lugar.

Un ejemplo de desarrollo en cuanto a la aplicación de las tecnologías de la educación y la comunicación TIC - a las actividades formativas, son las experiencias en el Instituto Tecnológico de Monterrey, donde ha sido notable la evolución del aula de clase. Se ha pasado por la clase presencial, la incorporación de tecnología en las aulas, la educación satelital, la educación en línea aprovechando la Web, hasta llegar en el 2010 a las innovaciones con m-learning [4].
En el 2007 en el Instituto se crearon ambientes con recursos para dispositivos móviles como audio, videos, ejercicios interactivos y actividades colaborativas, utilizándose artefactos como PDA-phones, teléfonos celulares, e-Books o BlackBerry [4].

El proyecto EDUMÓVIL [5] fue financiado en 2007 y 2008 por la Fundación Motorola, está dirigido a la aplicación del m-learning, para facilitar el aprendizaje de niños de escuelas primarias de la ciudad de Huajuapan de León (México). El propósito ha sido cubrir temas que presenten dificultad en el aprendizaje, por medio de un conjunto de juegos desarrollados para ser ejecutados en dispositivos móviles. Para esto se creó un proceso de desarrollo de juegos denominado GDP (Game Development Process), propuesto por el Grupo de Investigación de Tecnologías Aplicadas a la Enseñanza -GITAE- de la Universidad Tecnológica de la Mixteca (UTM).

Sobre este tema de los juegos en dispositivos móviles, existe un referente de la Facultad de Informática de la Universidad Complutense de Madrid. Allí el proyecto "Juegos Educativos en Dispositivos Móviles" se desarrollo dentro del concepto de ámbito del aprendizaje electrónico, combinando las ventajas de dos de sus categorías: el juego educativo y el aprendizaje móvil. Tal combinación hace posibles nuevas rutas y paradigmas en el diseño y creación de desarrollos como los videojuegos [6]. Este proyecto se desarrolló con base a dos plataformas: eAdventure y Android. El Software de e-Adventure está bajo licencia Open Source y se puede descargar de Internet, junto con manuales y tutoriales detallados.

Un prototipo WAP (Wireless Application Protocolprotocolo de aplicaciones inalámbricas) consistente en la implementación de un curso virtual inglés [7]. Para la construcción de este prototipo se desarrollaron modelos de casos de usos, y modelado de relaciones dominios, para un enfoque global de la solución. En el desarrollo se utilizaron herramientas de protocolos abiertos y software libre, como HTML y PHP. Algunas de sus funcionalidades son las siguientes:

- Despliegue de los contenidos académicos para usuarios registrados.

- Registro de estudiantes o usuarios.

- Obtención de contenidos programados dentro del portal WAP

- Evaluaciones en línea.

- Administración de la base de datos a través de la Web.

En la Universidad de Córdoba (España), se desarrollo un proyecto sobre incorporación de tecnologías mlearning a la docencia. El objetivo fue evaluar la utilización de distintos recursos de m-learning para la docencia de dicha institución. Concretamente se trabajó con PDAs (Personal Digital Assistant) y UMPCs (Ultra Mobile Personal Computer).

Proyecto MOBllearn [8] es una iniciativa cofinanciada por la Comisión Europea y la National Science

J. E. Parra. "Sistema de evaluación cualitativa a través de dispositivos móviles". Ing. USBMed, Vol. 3, No. 2, pp. 22-30. ISSN: 2027-5846. Julio-Diciembre, 2012. 
Foundation de los Estados Unidos, con los objetivos de diseñar una arquitectura que permita integrar los dispositivos móviles en entornos virtuales de aprendizaje, desarrollar aplicaciones de tipo cooperativas para dispositivos móviles, definir modelos teóricos de enseñanza y diseñar modelos de formación y contenidos para entornos que integren tecnologías móviles.

Otro proyecto que merece destacarse es ClayNet [9]. Es una plataforma de aprendizaje que se aplica tanto en procesos de apoyo a la educación presencial como en el aprendizaje en línea, con el agregado importante de poder ser adaptado y personalizado para cada estudiante. Su arquitectura se basa en servicios Web e integración de portlets (componentes modulares gestionadas y visualizadas en un portal web). El propósito fundamental es permitir el acceso de los usuarios desde un dispositivo móvil para el estudio e interacción con los recursos de aprendizaje.

En otro proyecto donde se evidencia la aplicación del software libre, es Mobile Learning EOI, desarrollado y aplicado en la Escuela de Organización Industrial, en cuya estructura se integran Android, Google, Moodle [10].

Teniendo Android como sistema operativo, se explotan recursos poderosos de Google Apps para el trabajo colaborativo, como correo, chat, sites, docs, debates, calendarios, blogs, grupos, en conexión con una plataforma educativa de Moodle, con el agregado de poder ser gestionada desde un dispositivo móvil. Este proyecto se enmarca dentro del Plan $2020 \mathrm{EOI}$, donde un objetivo prioritario es la apuesta por el conocimiento abierto y la integración de tecnologías de software libre.

En cuanto a su impacto y realización, dentro del proceso de formación de la EOI se ha dotado a más de 400 estudiantes de un dispositivo Smartphone, con sistema operativo Android (software libre) como parte de su programa formativo.

Una aplicación de los móviles en la construcción de una didáctica, se observa en [11], se describe un interesante proyecto didáctico que utiliza tres itinerarios didácticos desarrolladas sobre la plataforma Moodle (Open Source software) y englobaron el uso de agendas electrónicas o PDA (HP IPAQ Pocket PC 3715) y dispositivos GPS (Garmin eTrex).

En el primer itinerario el alumno, a través de la adopción de un rol de explorador para buscar un tesoro en un territorio desconocido, aprenda a orientarse geográficamente, a la vez que se familiariza con la utilización de los dispositivos móviles (PDA y GPS), Asimismo, en el segundo itinerario el alumno adopta el rol de arqueólogo y tendrá que sumergirse en la historia e influencia extranjera en esa región, también con dispositivos móviles y en el tercero, el alumno adopta el rol de artista y debe realizar una guía turística en la misma región.
La iniciativa $K-N e c t$, es otra aplicación del aprendizaje móvil. En cinco escuelas de secundaria de Carolina del Norte (EE.UU) los estudiantes con dificultades para aprender matemáticas están utilizando el programa $K$ Nect, ellos reciben los problemas de álgebra en el dispositivo móvil (smartphones), suben las respuestas a un blog comunitario en la Web y comentan los resultados con los compañeros y su profesor. Además, la intención es fomentar el trabajo en equipo y aprovechar las herramientas de audio y video, es por esto que resuelven los problemas por grupos y graban sus explicaciones para que sus compañeros puedan observarlas en el blog, intercambiando los roles de quien enseña y quien escucha [5].

Como este existen proyectos pilotos financiados por compañías de nuevas tecnologías, como es el caso de K-Nect o Nokia, con el proyecto Text2teach en Filipinas.

En Gran Bretaña está en marcha el proyecto MoLeNET, donde participan 115 colegios y 29 escuelas. "A través de diferentes iniciativas, el proyecto se basa en explorar las posibilidades de los soportes electrónicos móviles en general, tanto teléfonos como PDAs, reproductores de mp3 y mp4, videojuegos y netbooks, educando a los profesores en el potencial de estas herramientas con el fin de ampliar las habilidades del alumnado y generar más interés en el aprendizaje a través de actividades interactivas" [5].

\section{ANÁLISIS DEL SISTEMA}

Pruebal es un sistema para el diseño, aplicación y despliegue de pruebas de evaluación y resultados. El aplicativo consiste en un servicio Web y un modelo de cliente que accede a dicho servicio desde dispositivos móviles.

En el servicio Web se implementan los métodos necesarios para permitir y controlar la conexión y evaluación de los usuarios desde los móviles, así como el acceso a las notificaciones, adaptándolos a las características particulares del dispositivo utilizado. Desde el cliente móvil se ejecutan los métodos necesarios para el acceso al servicio web.

Es de anotar que el sistema se probó en dispositivos celulares de discreta gama (Sony Ericsson W302), asequibles sin altos comerciales y en otros dispositivos modernos, específicamente (Black Berry Curve 9300).

Con respecto a la validación de los resultados, el sistema se sometió a pruebas en tiempo real con usuarios de los dispositivos mencionados. Aun siendo un prototipo, de manera cualitativa se pudo verificar la buena apreciación e impacto que despertó en las experimentaciones. En general, dos aspectos fueron resaltados por los participantes en las pruebas:

a) La utilidad del proyecto, ya que cubre la necesidad de movilidad de las personas y está en coherencia con la cultura digital de los estudiantes jóvenes. 
b) Su potencial para ser incorporado en proyectos de educación virtual.

En las pruebas no se consideraron análisis cuantitativos ya que su naturaleza implicó técnicas de exploración bibliográfica y de aplicación de tecnologías para la Web móvil.

\subsection{Descripción del sistema}

La delimitación de Pruebal tiene las siguientes condiciones:

1) Existencia de un objeto de evaluación. A través del dispositivo móvil el estudiante recibirá instrucciones sobre el material de estudio, la ubicación del material, la fecha y hora de aplicación de la prueba, las condiciones de aceptación y los mecanismos para la interacción.

2) Como el estudiante responderá de acuerdo con unas opciones homologables a escala de Likert, las preguntas deben tener las siguientes orientaciones:

- Enunciados para análisis de respuestas argumentativas.

- Enunciados para análisis de respuestas interpretativas.

- Enunciados para análisis de respuestas propositivas.

3) Por las condiciones tecnológicas de los dispositivos móviles, en cuanto al reducido tamaño de las interfaces y la minimización de funciones incorporadas en los teclados, las preguntas deben formularse con texto condensado, con la intención de resumir al menor número de palabras. El texto debe escribirse para su rápida comprensión, evitándose proposiciones argumentativas extensas.

4) En un formulario para la Web, el profesor diseña la estructura de la prueba, la cual consiste en la escritura de los enunciados de las preguntas, la escritura de las opciones de respuesta para la homologación con la escala de Likert y el tiempo de respuesta.

Las preguntas se plantean para que el estudiante responda de acuerdo con cinco opciones, cada una de las cuales tiene un peso distinto, que internamente será interpretado por el software para la clasificación siguiente:

Tabla No 1. Escalas y valores para el diseño de las preguntas

\begin{tabular}{|c|l|}
\hline Escala & \multicolumn{1}{|c|}{ Valoración } \\
\hline \multirow{2}{*}{ A } & $\begin{array}{l}\text { La respuesta cumple de manera } \\
\text { satisfactoria con el objeto de } \\
\text { evaluación. } \\
\text { Respuesta totalmente correcta. }\end{array}$ \\
\hline \multirow{3}{B}{} & $\begin{array}{l}\text { La respuesta cumple de manera } \\
\text { satisfactoria con el objeto de } \\
\text { evaluación, pero agrega u omite } \\
\text { características importantes. }\end{array}$ \\
\hline
\end{tabular}

\begin{tabular}{|c|c|}
\hline Escala & Valoración \\
\hline & $\begin{array}{l}\text { Respuesta correcta } \\
\text { inconsistencias }\end{array}$ \\
\hline C & $\begin{array}{l}\text { La respuesta no es satisfactoria } \\
\text { aunque cumple parcialmente con } \\
\text { algunos atributos relevantes del } \\
\text { objeto de evaluación. } \\
\text { Respuesta que no es correcta ni } \\
\text { incorrecta }\end{array}$ \\
\hline D & $\begin{array}{l}\text { La respuesta no es satisfactoria y no } \\
\text { cumple con los atributos del objeto } \\
\text { de evaluación, aunque alcanza a } \\
\text { satisfacer algunos. } \\
\text { Respuesta incorrecta pero con } \\
\text { aciertos. }\end{array}$ \\
\hline$E$ & $\begin{array}{l}\text { La respuesta no es satisfactoria y } \\
\text { cumple atributos del objeto de } \\
\text { evaluación, los omite, los contradice } \\
\text { o no los comprende. } \\
\text { Respuesta totalmente incorrecta. }\end{array}$ \\
\hline
\end{tabular}

5) Existen bases de datos de profesores, estudiantes, pruebas y preguntas, para gestionar el acceso, el diseño, la aplicación y la gestión del sistema.

6) El sistema tiene opciones para su aplicación grupal o individual. Esto significa que las pruebas se pueden aplicar masivamente a grupos 0 individualmente a estudiantes seleccionados.

\subsection{Formulario para creación de preguntas}

La creación de preguntas se realiza en un formulario Web en función de los siguientes campos: número de la pregunta -NP-, peso de la pregunta -PP- (rango de 1 a 10), enunciado -E-, opciones de respuesta -OR-, valoración de cada opción -VO- (en el rango A, B, C, D, E).

La función del PP jerarquiza las preguntas, según lo decida el profesor. Varias preguntas pueden tener el mismo PP, pero ninguna de ellas puede quedar sin PP asignado. Como reglas de negocio, NP puede crecer hasta 20 y E puede tener hasta 40 palabras, por las condiciones tecnológicas y de usabilidad que implica m-learning lgualmente, la dimensión de las OR pueden ser hasta 30 palabras.

La VO se hace de acuerdo con la tabla No 1. A cada opción se le asigna una de las valoraciones y no puede haber opciones con igual valor. Esto significa que en tiempo de creación de la pregunta se le están dando al software los parámetros para la respectiva evaluación. La opción correcta tienen una valoración de $\mathrm{A}$ y el resto de valores se asignan jerárquicamente, dependiendo de la coherencia con respecto al enunciado.

Esto quiere decir que el Sistema Pruebal de manera cualitativa interpreta cada una de las posibles opciones y considera que dentro de las no acertadas hay unas más aproximadas que otras a la respuesta correcta.

El actor de esta plantilla es el profesor. Redacta los enunciados de pregunta, redacta las opciones y a cada 
opción le asigna una valoración en el rango de A hasta E.

Al estudiante le llegan las preguntas y las opciones. El estudiante elige una de las cinco opciones e internamente el programa la califica de acuerdo con la valoración asignada en la plantilla.

El profesor planea un número de preguntas superior al que recibirá el estudiante. Esto es, cada prueba tiene un banco de preguntas, de las cuales el programa escogerá aleatoriamente las que le llegarán al estudiante.

\subsection{Funcionalidad del sistema}

El sistema cumple con las siguientes funciones:

1) Aplicación de un servicio Web para programación de la prueba, diseño de preguntas y notificación a los dispositivos móviles de los estudiantes sobre la prueba programada. Envío de pruebas a los dispositivos y registro de las respuestas de los estudiantes.

2) La evaluación es interactiva, lo que significa que las preguntas no se despliegan en lista, sino que en la medida que el estudiante responde, se van mostrando en su dispositivo móvil.

3) En el dispositivo móvil del estudiante, se muestra el enunciado de la pregunta con un menú de cinco opciones de respuesta (cinco ítems). El estudiante elige uno de ellos y el software internamente lo homologa a la escala de Likert diseñada.

4) Las preguntas se emiten de acuerdo con un procedimiento aleatorio, en cuanto al orden de las opciones. Es decir, a estudiantes distintos les llega la misma prueba con preguntas distintas y con opciones de respuesta en orden diferente, en caso de preguntas iguales.

5) Terminada la totalidad de la evaluación, el software devuelve en el dispositivo móvil del estudiante y en su correo electrónico, los resultados de la prueba, comparando cada respuesta del estudiante con la respuesta acertada que almacenó el profesor.

6) El modelo de Pruebal permite que a los estudiantes le lleguen automáticamente a sus dispositivos móviles, los resultados con valoraciones cuantitativas y cualitativas de cada pregunta del examen.

7) En bases de datos con acceso a los profesores desde la Web, quedan registros de fecha, hora y resultados de las pruebas aplicadas.

8) El sistema queda reiniciado para una siguiente prueba. No es posible que a un estudiante le lleguen varias pruebas a la vez, sino que el software envía la evaluación siguiente a un estudiante, solo cuando cerró la última presentada.

\subsection{Expresión de los resultados}

Al estudiante le llega una valoración por cada pregunta. De acuerdo con el siguiente criterio se hace la evaluación, sabiendo que para cada pregunta el estudiante respondió $A, B, C, D \circ E$ :

Tabla No 2. Relación de conceptos y valoraciones

\begin{tabular}{|c|l|}
\hline Valoración & \multicolumn{1}{|c|}{ Concepto emitido } \\
\hline A & Respuesta totalmente correcta \\
\hline B & $\begin{array}{l}\text { Respuesta correcta pero con } \\
\text { inconsistencias }\end{array}$ \\
\hline C & $\begin{array}{l}\text { Respuesta que no es correcta ni } \\
\text { incorrecta }\end{array}$ \\
\hline D & $\begin{array}{l}\text { Respuesta incorrecta pero con } \\
\text { aciertos }\end{array}$ \\
\hline E & Respuesta totalmente incorrecta \\
\hline
\end{tabular}

El resultado general de la prueba se logra con la siguiente estructura:

Cada valoración tiene asociado un número en la escala de 1 a 5 . Esto permite hallar la media de la prueba, hecha la conversión es posible plantear procesos estadísticos para hallar el resultado de la prueba.

Cada pregunta tiene un peso asociado, este parámetro se utilizará en la obtención estadística del resultado de la prueba.

\begin{tabular}{|c|c|c|c|c|}
\hline & & 3 & & \\
\hline 5 & 4 & & 2 & 1 \\
\hline A & B & & D & $\mathrm{E}$ \\
\hline & & C & & \\
\hline
\end{tabular}

Fig. 1. Escala de Likert

Luego el resultado final se puede expresar en los siguientes términos:

a) Porcentaje de cumplimiento de logros: El resultado se puede expresar porcentualmente.

b) Evaluación en un rango de 1 a 5 .

c) Evaluación descriptiva de acuerdo con la siguiente homologación:

Tabla No 3. Criterios para el resultado final de la prueba

\begin{tabular}{|l|l|}
\hline Intervalo & \multicolumn{1}{c|}{ Concepto } \\
\hline 1.0 & Prueba reprobada \\
\hline $1.1-2.5$ & Prueba reprobada con algunos aciertos \\
\hline $2.6-3.5$ & $\begin{array}{l}\text { Prueba en suspenso. NI aprobado, ni } \\
\text { reprobada }\end{array}$ \\
\hline $3.6-4.9$ & Prueba aprobada con algunos desaciertos \\
\hline 5.0 & Prueba aprobada \\
\hline
\end{tabular}

\subsection{Análisis y diseño}

Los procesos de definición del sistema, análisis de requisitos, modelado de la solución y validación de requerimientos, se hicieron aplicando técnicas de UML. 
En las figuras 2, 3, 4 y 5, se muestra el modelado del sistema y los subsistemas.

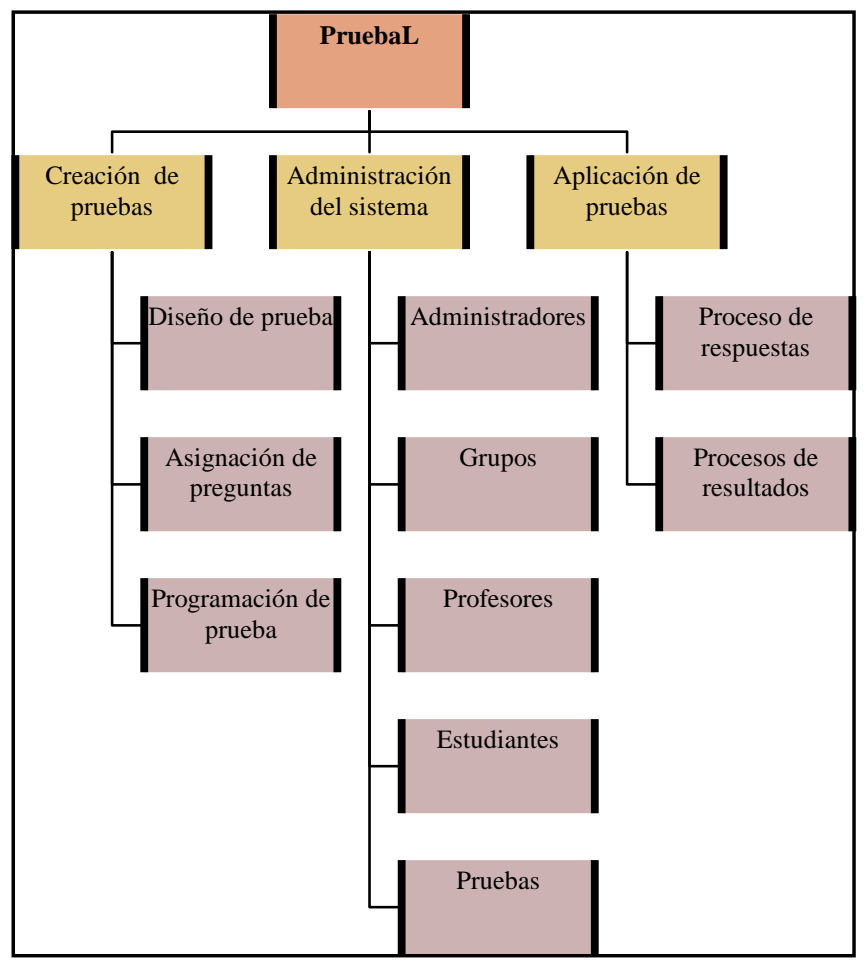

Fig. 2: Sistema global y subsistemas de PruebaL

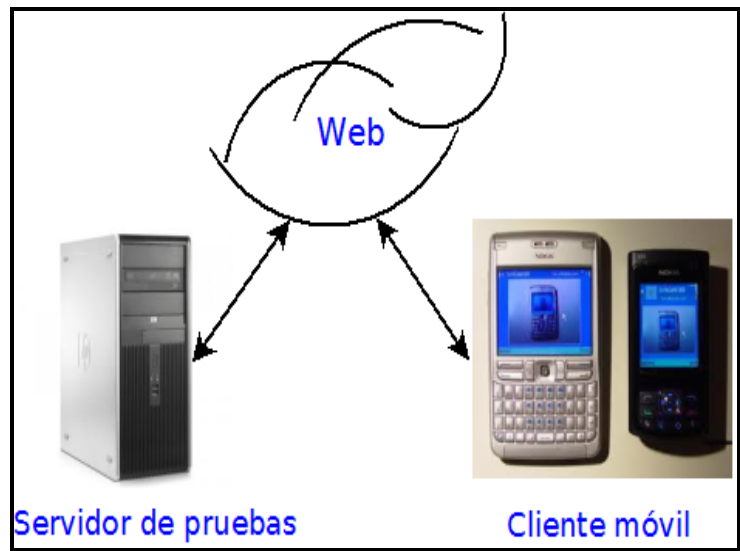

Fig. 3. Modelo global de PruebaL

El listado de requisitos que se logró estructurar en un modelo como parte del proceso de desarrollo de software, es el siguiente:

R1: Diseño de pruebas

R2: Creación de preguntas

R3: Programación de pruebas

R4: Edición de pruebas

R5: Visualización de pruebas

R6: Envío de pruebas

R7: Confirmación de notificación de pruebas

R8: Aplicación de pruebas

R9: Gestión del administrador

R10: Gestión del profesor

R11: Gestión del estudiante

R12: Gestión del grupo
Algunos de los diagramas UML implementados son los siguientes:

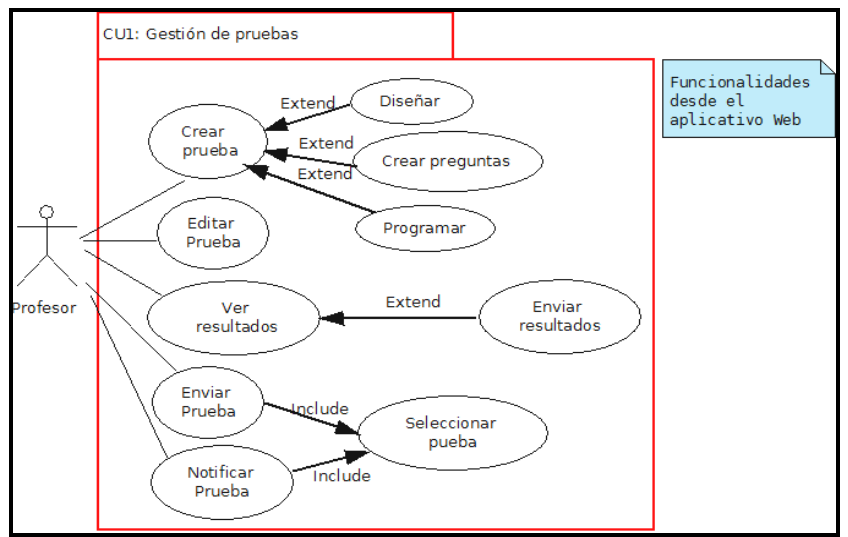

Fig. 4: Caso de uso de gestión de pruebas

Nombre del caso de uso: CU1 Servicio de pruebas

Pre-condiciones: Cosas que deben ser verdad antes que el caso de uso pueda suceder.

a. La plantilla debe estar habilitada para diseñar la prueba

b. Estudiantes y profesores deben estar matriculados antes de enviar notificaciones de pruebas y preguntas de las pruebas.

c. La notificación de una prueba y la aceptación del usuario, preceden a la aplicación de la prueba.

d. Los resultados de una prueba se envían al correo electrónico del estudiante o a su móvil. El envío es decisión del profesor u obedece a la solicitud de un estudiante.

e. Una prueba se puede ver, editar o eliminar, solo si el diseño está terminado.

f. Deben existir métodos para homologar los resultados a escalas de Likert.

Post-condiciones: Cosas que deben ser verdad después de que el caso de uso se haya realizado.

a. Las pruebas aplicadas quedan grabadas como plantillas llenas.

b. Los resultados de las pruebas deben quedar grabados en formato de escalas de Likert.

c. Los estudiantes reciben notificaciones, pruebas y resultados de las pruebas.

Objetivo: Gestionar los procesos del servicio Web de pruebas.

Descripción: Las funcionalidades de la gestión y resultados de las pruebas, habilitan al profesor para el diseño, edición, aplicación y almacenamiento de las pruebas y los resultados. 


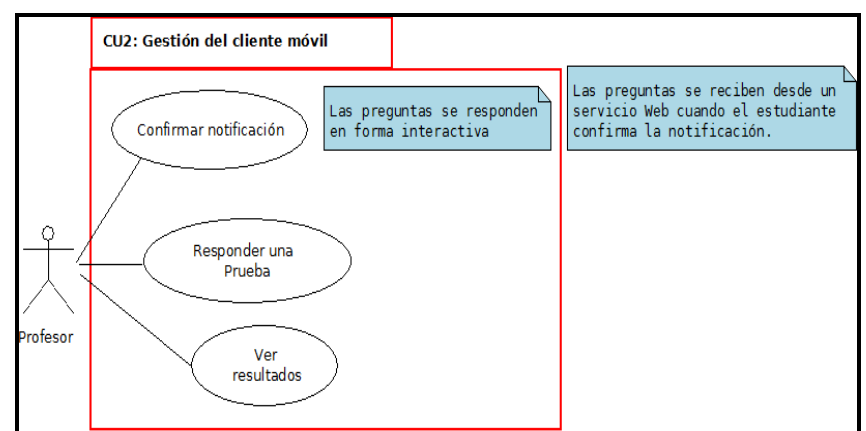

Fig. 5: Caso de uso de gestión del cliente móvil

Nombre del caso de uso: CU2 Gestión del cliente móvil

Pre-condiciones: Cosas que deben ser verdad antes que el caso de uso pueda suceder.

a. Las pruebas fueron diseñadas en el servicio Web.

b. La primera pregunta llega al dispositivo móvil, solo cuando el estudiante respondió a la notificación.

c. La siguiente pregunta llega solo cuando el estudiante respondió a la anterior.

Post-condiciones: Cosas que deben ser verdad después de que el caso de uso se haya realizado.

a. El estudiante respondió a las preguntas desde el dispositivo móvil

b. El estudiante recibió los resultados de las pruebas en formato de escalas de Likert.

Objetivo: Gestionar los procesos de aplicación de pruebas desde los dispositivos móviles de los estudiantes.

Descripción: Las funcionalidades del cliente móvil habilitan al estudiante para responder a las pruebas y para recibir notificaciones y resultados.

\subsection{Implementación}

La implementación de PruebaL requirió de los siguientes requisitos técnicos:

- Un servidor Web para hospedar bases de datos de prueba, estudiantes, dispositivos móviles y resultados.

- Aplicativo Web con formularios para el diseño y gestión de las pruebas.

- Interfaces de comunicación del aplicativo Web con los dispositivos móviles, para la aplicación de las pruebas.

- Herramientas de J2ME y JDBC de la familia de Java, para tener acceso a las bases de datos desde los dispositivos móviles, para lograr que la prueba sea interactiva. Las bases de datos se gestionarán con MySQL y los formularios para el diseño y gestión de pruebas, se desarrollaran con PHP.
Tipos de dispositivos móviles:

Pruebal requiere de dispositivos móviles con tecnología WAP - Wireless Application Protocol -, protocolo para comunicaciones y aplicaciones inalámbricas. El estándar WAP soporta la mayoría de las redes inalámbricas, incluyendo CDPD, CDMA, GSM, PDC, PHS, TDMA, FLEX, ReFLEX, iDEN, TETRA, DECT, DataTAC y Mobitex y es soportado por todos los sistemas operativos.

Los WAP que utilizan pantallas de baja gama y tienen acceso a Internet utilizando micronavegadores con archivos de pequeño tamaño, adaptables a las restricciones de memorias pequeñas, bajo ancho de banda y limitaciones funcionales que tienen los dispositivos que utilizan este estándar.

Aunque WAP soporta HTML y XML, el lenguaje WML (una aplicación XML) proporciona especificaciones para pantallas pequeñas y con navegación táctil sin teclados físicos.

El propósito es que a PruebaL se pueda acceder desde teléfonos celulares de uso común que tenga la capacidad de conexión a internet.

\subsection{Pruebas del sistema}

El sistema se probó utilizando teléfonos celulares de tecnología SonyEricsson Modelo W302, con memoria de 15.9 Mbytes y BlackBerry Curve 3G 9300, con conexiones HSDPA y Wi-Fi para un rápido acceso a Internet.

El plan de pruebas incluyo la matrícula de múltiples usuarios administradores, profesores y estudiantes. Se diseñaron, programaron, notificaron y enviaron exámenes de las materias bases de datos, algoritmos y economía básica.

El servicio Web se alojó en un servidor libre $\mathrm{x} 10 \mathrm{Hosting}$, el cual ofrece a los usuarios una configuración a través de la herramienta cPanel 11.

La aplicación se conectó con servicio de mensajería SMS a través de un Web Service administrado por una empresa privada (http://www.elibom.com).

\section{CONCLUSIONES}

Las metodologías, técnicas y tecnología para el desarrollo de software, no tienen modificaciones relevantes cuando se crean soluciones para dispositivos móviles, independiente de los requerimientos funcionales que deben considerar factores de usabilidad, dadas las limitaciones por el tamaño reducido de los dispositivos y de restricciones para la navegabilidad. Además, con respecto a los requisitos no funcionales, debe considerarse aspectos relacionados con la privacidad de las redes inalámbricas, el ancho de banda, las especificaciones tecnológicas de los dispositivos y el costo de las comunicaciones.

Con respecto al m-learning, las potencialidades de las redes inalámbricas, la proliferación de la tecnología 
móvil y la cultura digital de las personas, determinante para necesidades de acceso a las comunicaciones sin restricciones de localización o temporalidad, hacen viables propuestas para procesos de aprendizaje y evaluación a través de dispositivos móviles. Esto justifica emprender proyectos para el desarrollo de software dentro de los conceptos de Mobile Web y mensajería SMS.

Una última conclusión está relacionada con la evaluación cualitativa. Esta es viable aplicarla en entornos digitales, si se diseñan patrones que permitan mapear las respuestas de los estudiantes con valoraciones predefinidas y graduadas con escalas como la de Likert.

Dos recomendaciones quedan como resultado del proyecto presentado: el análisis de sistemas para dispositivos móviles debe considerar de manera muy completa los alcances de la tecnología móvil elegida, en cuanto a su accesibilidad, disponibilidad comercial y pertinencia; los proyectos de educación virtual y de aplicación de tecnologías de la información y la comunicación en educación presencial, tener presente alternativas para el m-learning, en consideración del impacto y penetración de las redes inalámbricas y los dispositivos móviles.

\section{REFERENCIAS}

[1] Innovación en Servicios Empresariales Avanzados ISEA-, MOBILE LEARNING, Análisis prospectivo de las potencialidades asociadas al Mobiille Learniing. Madrid: Plan Avanza. 2009.

[2] I. Fernández, (sf). Construcción de una escala de actitudes tipo Likert. Online [Enero. 2010].
[3] M. Ramírez. "Dispositivos móviles para ambientes virtuales: implicaciones en el diseño y la enseñanza," Redalyc, Vol. 8, No 9, pp. 82-96, Diciembre 2008.

[4] A. M. García-Valcárcel, "Modelos y estrategias de enseñanza". Online [Junio. 2009].

[5] G. G. Castillo, C. A. Fernández \& R. Ruíz. "Evolución del Proceso de desarrollo de video juegos en la iniciativa académica EDUMOVIL", in VII Jornadas Iberoamericanas de Ingeniería del Software e Ingeniería del Conocimiento. Universidad Tecnológica de la Mixteca. 2010.

[6] J. M. Cuevas, G. M. Pérez \& A. Villoria. "Juegos educativos en dispositivos móviles". Madrid: Universidad Complutense de Madrid, Facultad de Informática. 2009.

[7] M. Díaz, J. Garrido \& D. Heredia. "Prototipo de Mlearning". Revista Investigación y Desarrollo en TIC, No 1, pp. 38-46. 2010.

[8] M. Sánchez \& Y. Vicente (sf). M-learning: un nuevo paradigma en teleeducación. Laboratorio de software de telecomunicaciones. Online [Noviembre. 2010].

[9] A. Velasco, J. Carabias, M. A. Conde \& F. J. García. "ClayNet: Adaptación de contenidos en m-learning". Salamanca (España), Universidad de Salamanca. 2007.

[10] Escuela de Organización Industrial (sf). Plan EOI 2020. Madrid: Autor.

[11] J. M. Correa, A. Ibañez \& E. Jiménez. "Lurquest: Aplicación de tecnología m-learning al aprendizaje del patrimonio", Didáctica de las Ciencias Sociales, Vol. 50, pp. 109-123. 2006. 